\title{
La culture est-elle soluble dans la concentration économique?
}

\author{
Emmanuel Négrier - Nectart $\mathrm{n}^{\circ} 6,2018$, p.34-43
}

La culture est, de façon écrasante, une affaire privée en France. Sur la totalité de la production culturelle de référence telle qu'elle est recensée par le Département des études, de la prospective et des Statistiques du ministère de la Culture ${ }^{1}$, on estime à $18 \%$ la part de la production non-marchande (c'est à dire dont le prix représente moins de $50 \%$ du coût de production par un important niveau de financement public) et à $82 \%$ celle de la production marchande ${ }^{2}$. Mais si le secteur non marchand pèse d'un poids relativement limité, il occupe une place considérablement plus importante en termes d'orientation globale du domaine culturel. C'est le plus souvent lui qui assume les fonctions de pépinière, de défrichage et de soutien des esthétiques émergentes, celles-là même qui seront ensuite couronnées, ou non, par les réseaux, le marché et/ou l'institution. L'intervention publique, elle-même, se situe au carrefour entre les deux. Elle se justifie - depuis Keynes - par l'idée que le marché n'est pas en capacité d'offrir services et bien culturels dans des conditions satisfaisantes de qualité et de diversité sociales, artistiques et territoriale. Plus la culture procède des intérêts, moins elle est intéressante, pourrait-on dire. Depuis plus d'une décennie, alors que la culture ${ }^{3}$ marque globalement le pas en termes de chiffre d'affaires, des phénomènes de concentration se développent. A tout seigneur tout honneur, au carrefour entre culture et communication, entre contenus et contenants, se trouvent les GAFAM (Google, Amazon, Facebook, Apple, Microsoft). Leur rôle majeur provient non seulement de leur puissance économique, mais aussi de leur capacité de préemption des innovations qui fourmillent dans la galaxie des startup. On a récemment vu à quel point ces géants contemporains disposaient de la capacité, d'une main, à obtenir des pouvoirs publics des facilités d'installation qui faussent la concurrence, tout en contestant à ces mêmes autorités le plus élémentaire pouvoir en démocratie économique : l'ajustement de la fiscalité sur les espaces de profit. En outre, alors que les 87,1 milliards de chiffre d'affaires consolidé du domaine culturel en France correspondent à 597000 emplois ${ }^{4}$, les chiffres sont respectivement de 4,4 milliards et de ... 5000 emplois pour Amazon en France ${ }^{5}$. En culture, un emploi correspond donc à 145000 euros de chiffre d'affaires. Chez Amazon, il en faut 880000 . Une piste pour éclairer les stratégies de développement de l'emploi, sans même évoquer la fragilisation de toute la chaîne du livre, par exemple, qu'implique le tapis rouge déroulé devant elle. Face à ce phénomène, peut-on encore invoquer Joseph Schumpeter et son concept de " destruction créatrice ${ }^{6}$ sans s'interroger sur son efficience et son incidence dans des domaines aussi spécifiques que celui de la culture?

Les enjeux de la concentration dans le domaine culturel concernent plusieurs secteurs, trop différents pour que ce papier se donne pour objectif d'en faire l'analyse détaillée.

\footnotetext{
${ }^{1}$ Le périmètre que considère le DEPS est harmonisé à l'échelle européenne. Il comprend les sous-secteurs suivants : agences de publicité, arts visuels, architecture, audiovisuel, enseignement culturel, livre et presse, patrimoine et spectacle vivant.

2 Tristan Picard : Le poids économique de la Culture en France en 2015, Culture-Chiffres, DEPS 2017-1

${ }^{3}$ La culture est ici au singulier en tant que raccourci du domaine culturel, et non comme croyance à l'unicité ou à l'univocité de " la » Culture, dont le caractère pluriel est l'une des bases de notre raisonnement ici.

${ }^{4}$ Idem DEPS 2017

${ }^{5}$ source : AFP et L'Expansion.com, 20/2/2017

${ }^{6}$ Joseph A.Schumpeter, Capitalisme, Socialisme et Démocratie, Paris : Payot 1951
} 
Historiquement, la chronique a été d'abord défrayée par l'entrée en lice de Live Nation dans la gestion d'un festival (Le Main Square, à Arras) derrière laquelle - nous y reviendrons - se profilait la firme multinationale Clear Channel, opératrice de publicité, de média et de services urbains. Mais on a tôt fait de parler, dans le même secteur musical, de Fimalac, de Lagardère, voire de TF1 ou de Sony, comme caractéristiques de ces tendances. Dans le secteur de l'édition, la prise de contrôle des Éditions de la Martinière par Média Participations donne naissance à un troisième grand (derrière Hachette et Editis) au chiffre d'affaires d'environ 600 millions d'euros. Ces stratégies d'opérateurs culturels, auxquels on donne-parce qu'ils sont culturels - une dimension politique et morale, existent depuis longtemps. Leur singularité actuelle est sans doute liée au fait qu'il ne s'agit pas de prises de contrôle au sein d'un seul segment de marché, ou au sein du seul secteur marchand. Il s'agit aussi, et c'est ce qui va nous intéresser ici, de l'intervention de groupes marchands dans un des domaines où ils n'intervenaient pas jusque-là, soit que ces derniers touchaient à des compétences et activités distinctes, soit qu'il ne s'agissait pas même d'un secteur marchand. Ces nouveaux phénomènes témoignent donc de la remise en question de frontières jusqu'ici admises. Elle n'est pas l'apanage des seuls acteurs privés. La récente annonce d'un prélèvement sur la billetterie des scènes publiques pour améliorer le soutien au théâtre privé, issue d'une lecture du rapport Bonnell consacrée à ce sujet, a certes suscité une levée de boucliers ${ }^{7}$ des Scènes Nationales, mais il a aussi suscité de la part de Régine Hatchondo, directrice générale à la création artistique du ministère, ce commentaire : " / va falloir quand même penser à faire tomber le mur de Berlin entre vous et le théâtre privé ${ }^{8}$.

Nous voudrions montrer trois choses dans ce papier. La première est la coexistence de différentes formes de concentration à l'œuvre dans les filières culturelles. Pour cela, nous prendrons souvent l'exemple de la musique. Ensuite, nous examinerons les conséquences de l'inaction, et enfin les pistes de nature à redéfinir une action publique, adaptée à ce nouveau contexte.

\section{Comment?}

Le paysage de bataille que présente le secteur musical en France ne correspond pas à une seule forme de concentration économique. On peut au moins en distinguer trois. La première est financière, et permet à une firme de prendre le contrôle d'une autre tout en lui laissant une autonomie formelle, une identité de marque. Fimalac, dirigée par Marc Ladreit de Lacharrière, correspond bien à ce modèle, avec un chiffre d'affaire de près de 250 millions d'euros (en 2016) correspondant à un pôle divertissement (3S Entertainment) qui englobait, fin 2016, 101 salles de spectacles, 12 sociétés de production dont certaines, comme Miala, organisatrices de festivals. La préservation de l'identité des marques a pour objectif de ne pas rompre brutalement avec une personnalisation qui compte sur la valeur de ces entreprises, souvent artisanales et fragiles. Elle n'empêche pas les économies d'échelles, lorsqu'il s'agit pour la holding ou le pôle de faire adopter à ses filiales des services dont la société s'est rendue maîtresse, comme la billetterie par exemple. C'est le cas de MyTicket et de Tick \& Live pour Fimalac. Dans ce premier type de concentration, on peut trouver des acteurs qui se spécialisent sur plusieurs créneaux à la fois sans pour autant embrasser l'ensemble de la chaîne de valeur artistique. La stratégie de Fimalac prétend justement exclure le " $360^{\circ}$ " au

\footnotetext{
7 https://cultureveille.fr/scenes-nationales-sinsurgent-contre-reclamations-theatre-prive/

8 http://next.liberation.fr/theatre/2017/09/14/le-theatre-prive-veut-sa-part-du-public 1596425
} 
nom de la diversité artistique, tandis que le groupe Bolloré entend aller du développement artistique à leur production et leur tournée, jusqu'à leur valorisation dans les médias et dans I'industrie du disque, au travers de l'ensemble des ressources de Vivendi.

Le second type de concentration est horizontal, et se traduit par l'absorption de concurrents ou la duplication, dans le même sous-secteur, d'un événement, par exemple. On pense ici au groupe Live Nation et à ses déclinaisons d'un même festival dans plusieurs pays - comme le Lollapalooza - aux quelques 3300 artistes sous contrats qui tournent dans le monde, et notamment lors des 25500 concerts organisés annuellement par un groupe dont le chiffre d'affaires se situe, en 2016, à 7,5 milliards d'euros. Ce phénomène n'est pas si récent qu'il y paraît, puisque dans le secteur des musiques savantes, René Martin avait, à une échelle certes bien plus modeste, dupliqué son festival La Folle Journée dans plusieurs métropoles, tout en prenant la direction d'autres festivals en France (Saint-Chartier, La Roque d'Anthéron). On peut également évoquer le groupe Lagardère et sa stratégie de production d'artistes, à la manière dont la firme avait initié la gestion de la carrière de sportifs de haut niveau. Cette concentration sur un même créneau d'activité engendre des attentes d'économies d'échelle d'une plus grande ampleur que dans le cas précédent. Par exemple, la duplication d'un événement permet de mutualiser un certain nombre de coûts : graphisme, cachets artistiques en série, continuité de dispositif technique, communication, etc.

Le troisième type de concentration est vertical, et se traduit par l'absorption de clients ou de fournisseurs. On se rapproche du $360^{\circ}$, plus ou moins complètement, en capitalisant sur les interdépendances qui existent entre les risques initiaux (dans le développement d'un artiste, la création d'un label, d'un lieu culturel, etc.) et les bénéfices tirés de l'ensemble des exploitations possibles d'un artiste ou d'une œuvre. Nous retrouvons le cas de Fimalac, de Vivendi ou de Live Nation, déjà cités. On peut également mentionner le cas de Sony, du côté des équipements et du disque, dont l'investissement dans la production d'artistes est bien sûr lié à l'effondrement de la rentabilité de la musique enregistrée et du déplacement des sources de profit vers le spectacle.

\section{Pourquoi ?}

Un paradoxe surgit cependant. Ces opérations de rachat, de concentration ne se déroulent pas seulement dans une atmosphère économiquement morose. Elles n'ont en outre aucunement fait la preuve de leur rentabilité, au moins à court terme. Le résultat serait plutôt inverse. Comment dès lors expliquer que ces grandes manœuvres donnent à la fois l'image d'une ruée vers l'or et celle d'une bulle spéculative, dont les répercussions de l'éclatement pourraient affecter l'ensemble du domaine culturel ? Trois possibilités se présentent pour résoudre un tel paradoxe.

La première est un peu déprimante, car elle assure que nous serions myopes et que les grands opérateurs eux, seraient doués d'une hauteur de vue telle qu'ils sauraient anticiper ce que nous ne pourrions pas voir. Un scénario de moyen terme: débarrassés d'interventions publiques faussant le jeu et de concurrents anéantis par la course au gigantisme, quelques acteurs dominant une population toujours plus nombreuse et fragmentée de propositions artistiques interchangeables pourront fixer leur prix et l'imposer à des publics condamnés à en assumer l'inflation. On laisse le lecteur envisager toutes les implications d'un tel scénario. 
Deuxième possibilité, presque romantique, les acteurs concernés par ce vaste Monopoly réhabilitent la figure du sénateur de la III ${ }^{e ̀ m e}$ République, et les danseuses qui vont avec. La culture serait pour eux une manière de racheter l'image de sociétés dont les activités ne confèrent guère de prestige à leurs dirigeants: indice de cotation en bourse et activités immobilière pour Fimalac, armement puis littérature de gare pour Lagardère, publicité sur abribus pour Clear Channel... La culture répondrait donc pour ces capitaines d'industrie à une sorte de logique de l'honneur. On retrouve ici l'explication fournie, pour la presse, par Laurent Mauduit au sujet de l'engagement dans le capital du journal Le Monde de Xavier Niel, jadis inquiété pour ses frasques dans l'économie du peep-show et du sex-shop et condamné pour elles $^{9}$ : "sauver " Le Monde, c'est aussi se laver d'un passé peu glorieux. L'explication convaincrait s'il s'agissait ici ou là de moins gagner. On y croit moins s'il s'agit de valider des pertes durables.

La troisième explication insiste sur la nature des firmes qui investissent le champ. Nous l'avons noté, aucune n'est engagée dans le secteur culturel à titre exclusif. Quelle que soit la stratégie de concentration, la culture représente même le plus souvent une part minoritaire dans l'ensemble des activités des groupes: médias, équipements, finances, services urbains, immobilier, commerces de détail, sport. Par exemple, pour Fimalac, on estime à environ $30 \%$ le chiffre d'affaires généré par les activités culturelles dans l'ensemble de la holding. Cependant, cette part est, à l'échelle de ces groupes, parée de valeurs que n'ont pas d'autres investissements, autrement rentables pourtant. À l'image d'un mécène faisant fructifier, grâce à son engagement auprès d'un festival, les relations d'affaires dans les meilleures conditions qui soient, l'investissement dans la culture peut être un levier d'échanges pour d'autres activités du groupe. La culture n'est plus (simplement) une danseuse. Elle devient une entremetteuse. Et si cette activation des échanges fonctionne, c'est aussi parce que le bien culturel dont il est question (un festival, un catalogue d'artistes, un lieu culturel, etc.) renvoie à ce que Luc Boltanski et Renaud Esquerre ${ }^{10}$ appellent une forme de mise en valeur tendance : liée au caractère exceptionnel de sa création ou de son créateur, au récit et aux mythes qui l'entourent, aux investissements sociaux-libidinaux qui lui donnent chair. A condition de limiter les pertes dans l'économie du prix - ce que permet une concentration ouvrant sur des économies d'échelle - les gains sont substantiels dans l'économie (symbolique) de la valeur. Marc Ladreit de Lacharrière est transfiguré en sauveur de la culture française. Pour un peu, il incarnerait le retour du champion national néo-colbertiste dont le tournant néolibéral nous avait tristement privés.

\section{Laisser faire ?}

Pour répondre à cette question, il faut d'abord s'entendre sur l'impasse du non-agir. Ne rien faire se justifie sur trois bases possibles, diversement iniques. La première consiste à laisser la concurrence se développer sans entrave, en donnant au spectateur - consommateur final le soin d'arbitrer. C'est une position inique compte tenu, d'abord, de l'inégalité radicale du rapport entre offre et demande où entrent des capacités croissantes de séduction

\footnotetext{
${ }^{9}$ Laurent Mauduit, Main basse sur l'information, Paris : Don Quichotte, 2016

Cf. aussi : https://www.mediapart.fr/journal/france/201212/les-secrets-bien-gardes-de-xavier-niel

${ }^{10}$ Luc Boltanski, Renaud Esquerre, Enrichissement. Une critique de la marchandise, Paris Gallimard Essais, 2017
} 
commerciale. C'est d'autre part nier la philosophie de toute politique culturelle, qui consiste davantage à proposer aux gens ce qu'ils pourraient aimer que ce qu'ils croient aimer.

La deuxième justification est suiviste. Elle consiste à croire à la théorie du ruissellement ou de la percolation. Selon cette vision, les revenus colossaux engendrés par le business de la culture finissent par bénéficier aux acteurs émergents, au secteur non-marchand. C'est l'image des chorégraphes hip-hop, incapable de vivre de leurs créations, mais survivant grâce aux shows de variété et aux engagements publicitaires. Le problème est qu'une telle vision déséquilibre totalement l'ensemble du domaine culturel, et le rend globalement dépendant du " règne de la marchandise ", alors même que les ressources issues de ce ruissellement restent les plus limitées.

La troisième tentation du laisser-faire est celle du champion national, que les politiques devraient conforter parce qu'il est national, sans s'intéresser au contenu de la culture en jeu. On comprend aisément le caractère régressif et impraticable $d^{\prime}$ 'une telle argumentation.

\section{Agir ?}

$\mathrm{Si}$ les politiques publiques ne peuvent rester indifférentes à ces phénomènes de concentration, c'est parce qu'ils recèlent de véritables risques pour la diversité de la vie artistique et culturelle et pour la pluralité de destins de ce qu'agir en culture signifie. Sur le premier point, rappelons par exemple que le groupe iHeartMedia, qui coiffe Clear Channel et Live Nation, a été directement accusé de censure, en mettant à l'index de son empire radiophonique des propos jugés excessivement critiques à l'égard de Georges Bush, ou des œuvres musicales abordant des thématiques jugées indésirables après les attentats du 11 septembre 2001 (jusqu'à « Imagine » de John Lennon, ou « What a wonderful world » de Louis Armstrong ${ }^{11}$ ). La capacité de ces groupes à jouer des synergies entre différentes activités court également le risque de réduire la programmation artistique au développement de catalogues maison, certes diversifiés dans leurs styles et genres, mais soumis au même cursus économique. Les conséquences ne sont pas moins graves pour les différents acteurs, non marchands ou artisanaux, qui concourent aujourd'hui à la pluralité des trajectoires de reconnaissance artistique. Il en va de leur rôle comme des incidences globales sur l'emploi dans le secteur. Certaines activités sont déjà en train de péricliter, comme celle des promoteurs locaux de concerts ou d'artistes. D'autres sont menacées, comme celles des développeurs d'artistes, producteurs et labels indépendants. Or ils représentent à la fois un nombre considérable d'emplois, et une garantie de diversité artistique du secteur du spectacle et des musiques, entre autres.

Quelles sont les pistes d'action qui se présentent aujourd'hui à nous ? Parmi celles-ci, nous en proposons trois au débat. La première réside dans le renforcement de l'intervention publique aux côtés des acteurs les plus fragiles de ces filières, au besoin en sollicitant d'eux-mêmes qu'ils s'accordent sur leurs intérêts communs, par exemple à l'échelle régionale. Ce sont les tentatives d'organisation par le biais de Contrats de filière (Nouvelle Aquitaine), de réseau des musiques actuelles (Grand Est) ou de Solima ${ }^{12}$ (à plusieurs échelles territoriales) qui sont ici visés. Encore faut-il que ces initiatives ne soient pas conditionnées par une injonction à " changer de modèle économique " allant dans le sens d'une caporalisation du vaste monde

\footnotetext{
${ }^{11}$ Truitt, Eliza "It's the End of the World as Clear Channel Knows It". Chatterbox, 17-09-2001 Slate.com.

${ }^{12}$ Schémas d'Orientation et de développement des Lleux de Musiques Actuelles
} 
associatif, coopératif, informel qu'est aujourd'hui le monde de la culture à partir d'un seul patron : l'entreprise privée à but lucratif.

La deuxième piste touche aux formes de solidarité mécanique qui peuvent être le fruit de nouvelles règles du jeu. Par exemple, l'introduction d'un droit de suite au profit des acteurs culturels (souvent non marchands) qui ont pris les premiers risques auprès des artistes, mérite d'être discutée. Elle suscite des réserves légitimes, notamment quant au changement de mentalité du secteur non-marchand, tenté de jouer les détecteurs de pépites plutôt qu'éducateurs. Mais elle existe dans plusieurs disciplines sportives et a permis à de nombreux clubs amateurs de survivre. En culture, elle pose plusieurs problèmes, à commencer par la pluralité d'acteurs (programmateur, manager, développeur, centre socioculturel, etc.) qui, bien souvent, seraient l'équivalent du seul « club amateur » pour le sport. Et si le droit de suite n'existe pas en droit, il est souvent pratiqué en fait, par la reconnaissance que tel artiste doit à l'espace (social, territorial) où s'est développé son art. Tout la question est de savoir si ce " retour à la communauté », aujourd'hui informel, mérite d'être formalisé.

La troisième piste touche à la parafiscalité. Pour le spectacle vivant (secteur musical inclus), deux structures plus ou moins complémentaires et concurrentes existent : I'Association pour le Soutien au Théâtre Privé (ASTP), d'une part, et le Centre National de la Chanson, des Variétés et du Jazz (CNV). L'une et l'autre prélèvent, pour leurs sous-secteurs respectifs, une taxe de 3,5\% dont le produit est reversé en droit de tirage aux affiliés (pour la plus grosse part) et d'autre part à l'ensemble des acteurs de la filière selon des modalités définies au sein de commissions sectorielles. Compte tenu des interpénétrations logiques entre les champs respectifs des deux institutions, il est parfois difficile de distinguer un spectacle qui relève de I'une ou de l'autre institution. Le nombre de bénéficiaires des aides est bien sûr très différent (80 pour la première, la plupart parisiens ; 591 pour le second, beaucoup plus présent en région), mais une fusion semble digne d'une discussion, vers une sorte de Centre National du Spectacle, sur le modèle du CNC. De même, une taxation plus sensible aux effets indésirables de la concentration décrite plus haut devrait être débattue. Enfin, à l'heure où les notions de scènes, de places artistiques singulières sont en plein essor ${ }^{13}$, de tels outils méritent d'être mis au service du développement territorial, selon une gouvernance plus favorable à la diversité des acteurs marchands et non marchands de la culture.

${ }^{13}$ Gérôme Guibert, Guy Bellavance (dir.), La notion de " scène ", entre sociologie de la culture et sociologie urbaine : genèse, actualités et perspectives, Cahiers de Recherche Sociologique $n^{\circ} 57,2014$ 